\title{
Diagnostik von Binde- und Hornhauterkrankungen
}

\author{
Teil 1: Diagnostik des Tränenfilms und der Augenoberfläche
}

\section{Clinical Diagnostics for the Tear Film and the Ocular Surface}

\author{
S. Schrader, D. Finis, K. Spaniol, M. Roth, P. Ackermann, G. Geerling \\ Universitätsaugenklinik, Heinrich-Heine-Universität Düsseldorf
}

Das Krankheitsbild des trockenen Auges kann im Wesentlichen in 2 Formen unterteilt werden: Einen quantitativen und einen evaporativen Tränenmangel. Dabei macht der evaporative Tränenmangel mit einem Anteil von ca. zwei Dritteln den Großteil der Fälle aus.

Um Patienten mit trockenem Auge effizient behandeln zu können, ist eine genaue Diagnostik zur Evaluation der
Form und des Schweregrades entscheidend. Ziel dieses Beitrages ist es, einen Überblick über die wichtigsten Methoden zur Diagnose des quantitativen und evaporativen Tränenmangels sowie praktische Tipps zur Durchführung der Diagnostik zu geben, um sowohl die Form als auch den Schweregrad des trockenen Auges genau bestimmen und damit auch effizient und individuell behandeln zu können.

\section{Anamnese/Symptomfragebögen}

\author{
Standardisierte Fragebögen eignen sich sehr gut \\ für die Anamnese und zur Messung eines Therapie- \\ erfolges.
}

Vor jeder apparativen Diagnostik steht auch bei Oberflächenerkrankungen des Auges die Anamnese. Zur besseren Standardisierung der Anamnese stehen verschiedene Fragebögen zur Verfügung. Je nach Länge der Fragebögen werden dabei neben Symptomen auch die auslösenden Faktoren und die Auswirkungen auf die Lebensqualität abgefragt.

Um Wartezeiten effektiv zu nutzen, bietet ein strukturierter Fragebogen eine gute Möglichkeit zum Screening von Patienten mit potenziellen Oberflächenerkrankungen des Auges. Außerdem können die Symptome quantifiziert und ein Therapieerfolg im Verlauf besser beurteilt werden.

OSDI (Ocular Surface Disease Index) und DEQ (Dry Eye Questionnaire). Für klinische Studien empfiehlt sich die Verwendung der etablierten Fragebögen OSDI (Schiffman et al. 2000) oder DEQ (Begley et al. 2002), vor allem aufgrund der guten Quantifizierbarkeit des Schweregrades der Symptome.
IDEEL-Fragebogen (Impact of Dry Eye on Everyday Life questionnaire). Der IDEEL-Fragebogen scheint allerdings in der Erfassung von Auswirkungen des trockenen Auges auf die Lebensqualität dem OSDI überlegen zu sein (Grubbs et al. 2014).

SPEED (Standard Patient Evaluation of Eye Dryness). Vorteile kürzerer Fragebögen, wie des SPEEDFragebogens (Standard Patient Evaluation of Eye Dryness) hingegen liegen im geringeren Umfang und der leichteren Auswertbarkeit und erscheinen daher besonders attraktiv für die Verwendung in der täglichen Routine einer Augenarztpraxis.
Abkürzungen
BVA Berufsverband der Augenärzte
CLEK Collaborative Longitudinal Evaluation of Keratoconus
DEQ Dry Eye Questionnaire
DEWS Dry Eye WorkShop
FBUT Fluorescein-Break-up-Time
IDEEL Impact of Dry Eye on Everyday Life questionnaire
NIBUT nichtinvasive Break-up-Time
OCT optische Kohärenztomografie
OSDI Ocular Surface Disease Index
SPEED Standard Patient Evaluation of Eye Dryness 


\section{Tabelle 1}

Standardisierte Fragebögen zur Erfassung des Symptoms „trockenes Auge“.

\begin{tabular}{|c|c|c|c|}
\hline Fragebogen & & Vor-/Nachteile & Quelle \\
\hline OSDI & Ocular Surface Disease Index & $\begin{array}{l}\text { gute Quantifizierbarkeit des Schweregrades } \\
\text { der Symptome } \\
\text { - etabliert } \\
\text { - für klinische Studien geeignet }\end{array}$ & Schiffman et al. 2000 \\
\hline DEQ & Dry Eye Questionnaire & $\begin{array}{l}\text { - gute Quantifizierbarkeit des Schweregrades } \\
\text { der Symptome } \\
\text { - etabliert } \\
\text { - für klinische Studien geeignet }\end{array}$ & Begley et al. 2002 \\
\hline IDEEL & $\begin{array}{l}\text { Impact of Dry Eye on } \\
\text { Everyday Life questionnaire }\end{array}$ & $\begin{array}{l}\text { - gute Erfassung von Auswirkungen des trockenen Auges } \\
\text { auf die Lebensqualität }\end{array}$ & Grubbs et al. 2014 \\
\hline SPEED & $\begin{array}{l}\text { Standard Patient Evaluation } \\
\text { of Eye Dryness }\end{array}$ & $\begin{array}{l}\text { - } \text { kurz } \\
\text { - geringer Umfang } \\
\text { - leichte Auswertbarkeit } \\
\text { - für den Einsatz in der täglichen Routine geeignet }\end{array}$ & Finis et al. 2014 \\
\hline $\begin{array}{l}\text { Fragebogen des Ressorts } \\
\text { „trockenes Auge“ vom } \\
\text { Berufsverband der } \\
\text { Augenärzte }\end{array}$ & Berufsverband der Augenärzte & $\begin{array}{l}\text { - einfache Erfassung der Anamnese } \\
\text { - Nachteil: Es kann kein Punktwert generiert werden } \\
\rightarrow \text { keine Verlaufsbeurteilung möglich }\end{array}$ & \\
\hline
\end{tabular}

Fragebogen des Ressorts „trockenes Auge“ vom Berufsverband der Augenärzte. Alternativ zu den oben genannten Fragebögen kann auch noch der Fragebogen des Ressorts „trockenes Auge“ vom Berufsverband der Augenärzte verwendet werden. Dieser eignet sich sehr gut zur Erfassung der Anamnese, hat aber den großen Nachteil, dass kein Punktwert generiert werden kann, der eine Verlaufsbeurteilung ermöglicht.

Die unterschiedlichen Fragebögen zeigt Tab. 1 im Überblick.

\section{Tests zur Messung der Tränensekretion und des Tränenvolumens}

\section{Schirmer- und Jones-Test}

Beim Schirmer-Test wird ein Lackmus- oder Filterpapierstreifen der Größe $35 \times 5 \mathrm{~mm}$ am abgerundeten Ende umgeknickt und an der Grenze vom temporalen zum medialen Drittel des Unterlids in den Bindehautsack eingehängt (Abb. 1). Anschließend werden die
Augen geschlossen und das Ergebnis nach 5 Minuten abgelesen.

Der Schirmer-I-Test wird immer ohne Lokalanästhesie durchgeführt. Auch wenn der Patient angewiesen wird, die Augen zu schließen, entstehen häufig Reizungen des Auges durch das Reiben des Filterpapiers. Hieraus resultieren deutliche Schwankungen des Tests. Um diese zu vermeiden, wurde der Schirmer-II- oder auch Jones-Test entwickelt, der ca. 1 Minute nach Lokalanästhesie analog zum Schirmer-I-Test für 5 Minuten durchgeführt wird. Dieser Test soll die Basissekretion der Tränendrüse widerspiegeln. Da die Lidkanten allerdings nicht mitbetäubt werden, wird hier auch eine gewisse Reizsekretion gemessen.

Der in wissenschaftlichen Arbeiten verwendete Test ist hauptsächlich der Schirmer-I-Test. Die Sensitivität des Tests liegt bei einem Grenzwert von < 5,5 mm bei $85 \%$.

Als normal werden Werte von $\geq 15 \mathrm{~mm}$ angesehen. Beim Schirmer-II-Test liegen die Werte ca. 40\% niedriger. Die Durchschnittswerte von Männern und Frauen unterscheiden sich nicht signifikant. 
Mit zunehmendem Alter finden sich niedrigere Werte beim Schirmer-Test, wobei keine Grenzwerte für verschiedene Altersklassen definiert sind (Definition and Classification Subcommittee of the International Dry Eye WorkShop 2007; Jacobi et al. 2006).

\section{Phenol-Rot-Test}

Alternativ zum Schirmer-Test kann der Phenol-RotTest verwendet werden. Dieser Test basiert auf dem gleichen Prinzip wie der Schirmer-Test, versucht aber, dessen Nachteile auszugleichen. So wird ein dünner Baumwollfaden in den Bindehautsack eingebracht. Dieser verursacht keine wesentliche Reizung, sodass eine Lokalanästhesie überflüssig ist.

Im Gegensatz zum Schirmer-Test wird bereits nach $15 \mathrm{~s}$ abgelesen, sodass das Ergebnis wesentlich schneller zur Verfügung steht. Bei einem Grenzwert von $12 \mathrm{~mm}$ bietet der Test eine Sensitivität von 56\% und ein Spezifität von $69 \%$. Es findet sich insgesamt eine hohe Übereinstimmung mit dem Schirmer-Test, jedoch zeigte sich in einer Studie bei 32\% der Patienten keine Übereinstimmung der Ergebnisse. Es werden in Zukunft weitere Studien nötig sein, um die Korrelation der beiden Verfahren genauer bestimmen zu können (Tomlinson et al. 2011).

\section{Meniskometrie}

Die Höhe des Tränenmeniskus am Ober- und Unterlid gibt direkt an, wie viel Tränenflüssigkeit beim nächsten Lidschlag zur Verfügung steht. Der Wert kann zunächst relativ einfach an der Spaltlampe über die Höhe des Spaltes gemessen werden. Die Methode ist jedoch relativ ungenau, und teilweise muss Fluorescein für eine ausreichende Visualisierung des Meniskus verwendet werden. Dies wiederum beeinflusst jedoch das Messergebnis durch die Zugabe von Flüssigkeit bzw. durch Reizung des Auges mit einer darauf folgend erhöhten Tränenproduktion.

Eine genauere Messung gelingt, wenn man ein Muster von schwarzen und weißen Streifen auf das Auge projiziert. Der Tränenmeniskus grenzt sich hierdurch deutlich besser ab. Die Bilder werden mit einer Kamera erfasst und computergestützt ausgewertet.

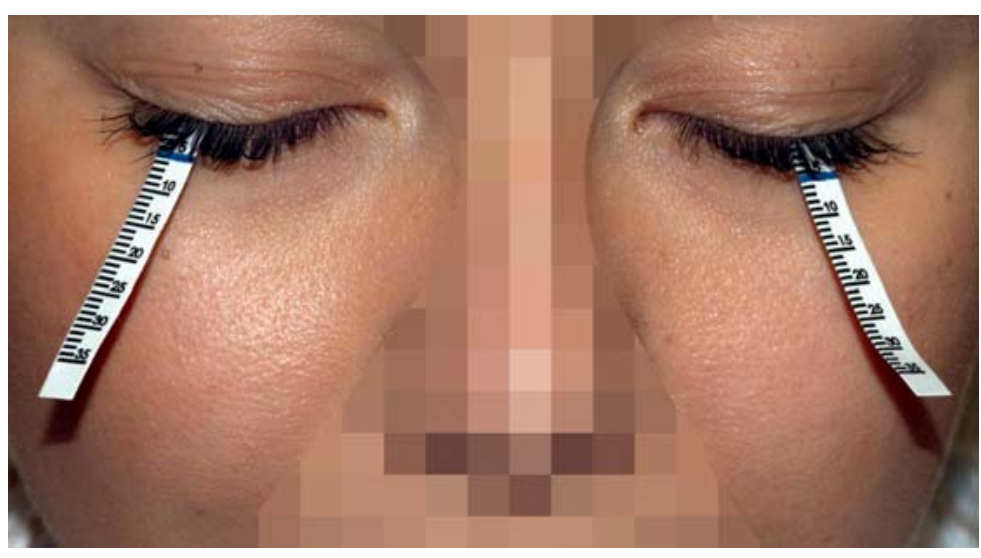

Abb. 1 Schirmer-Test.

Neuerdings kann man die Höhe des Tränenmeniskus auch mittels Spectral-Domain-OCT messen. Außerdem gibt es automatisierte Messgeräte, die den Tränenmeniskus mittels Placido-Ringen visualisieren.

Die Normwerte variieren je nach Verfahren. Insgesamt gelten Werte unter 0,2 mm als pathologisch (Brewitt u. Zierhut 2001). Die Höhe des Tränenmeniskus korreliert mit dem Schirmer-Test und steigt nach Applikation von Augentropfen oder Einbringen von Punctum-Okklusiven (Yokoi et al. 2004).

\section{Fluorophotometrie}

Die Fluorophotometrie quantifiziert den nach Eingabe von Fluorescein in den Bindehautsack zu beobachtenden Abfluss des Fluoresceins. Je mehr Tränen produziert werden bzw. abfließen, desto schneller wird der Farbstoff auch eliminiert.

Bei der Untersuchung wird zunächst durch ein Gerät die Autofluoreszenz des vorderen Auges erfasst. Anschließend wird eine bekannte Menge Fluorescein (z.B. $1 \mu \mathrm{l}$ ) in bekannter Konzentration (z.B. $2 \%$ ) in den Bindesack eingegeben und die Fluoreszenz über einen Zeitraum von 20 Minuten gemessen. Problematisch sind eine lange Untersuchungsdauer und Messfehler bei gestörter Epithelbarriere mit Aufnahme von Fluorescein in das Gewebe (Definition and Classification Subcommittee of the International Dry Eye WorkShop 2007). 


\section{Zielgerichtete Diagnostik}

Stellenwert der Tests zur Messung der Tränensekretion und des Tränenvolumens

- Standardtest zur Messung der Tränensekretion ist der Schirmer-I-Test.

- Die Höhe des Tränenmeniskus gibt an, wie viel Flüssigkeit beim nächsten Lidschlag zur Verfügung steht.

\section{Qualitative Untersuchungen des Tränenfilms}

\section{Tränenfilmaufrisszeit}

Die Tränenfilmaufrisszeit (Break-up-Time) ist der klassische Test zur Diagnose eines evaporativ trockenen Auges.

Die Stabilität des Tränenfilms ist von verschiedenen Faktoren abhängig, vor allem aber von der Integrität der Lipidschicht. Diese ist wiederum hauptsächlich von der Funktion der Meibom-Drüsen abhängig, weshalb eine reduzierte Aufrisszeit als Hinweis auf eine Meibom-Drüsen-Dysfunktion gewertet werden kann.

Zur Messung wird klassischerweise ein mit Kochsalzlösung getränkter Streifen Fluorescein in den unteren Fornix eingebracht. Anschließend wird der Patient gebeten, einige Male zu blinzeln und die Augen zu bewegen, damit sich der Farbstoff gut im Tränenfilm verteilt. Schließlich wird der Tränenfilm mittels Spaltlampe mit vorgeschaltetem Kobaltblaufilter im Bestrahlungsgang betrachtet. Der Patient wird angewiesen, zunächst natürlich zu blinzeln und dann die Augen aufzuhalten und geradeaus zu schauen. Es wird die Zeit vom letzten Lidschlag bis zum Aufreißen des Tränenfilms gemessen, was durch schwarze Flecken im Fluoresceinfilm erkennbar wird. Um möglichst die gesamte Kornea betrachten zu können, wird ein breiter Spalt in voller Höhe gewählt und zudem der Spalt über die Hornhaut bewegt.

Alternativ zu den Fluoresceinstreifen kann auch flüssiges Fluorescein mittels Mikropipette verwendet werden. Hierbei kommen Konzentrationen von 2-5\% und Volumina von 1-5 $\mu \mathrm{l}$ zum Einsatz.

Typischerweise wird nach der Fluorescein-Break-upTime (FBUT) die Anfärbbarkeit der Hornhaut und Bindehaut betrachtet. Da das Fluorescein in die Hornhaut und Bindehaut aufgenommen wird und so mit der Zeit eine Anfärbbarkeit, z.B. von Epitheldefekten, schlechter vom intakten Gewebe abzugrenzen ist, empfiehlt es sich, die Prozedur zunächst an einem Auge durchzuführen und erst danach den Farbstoff in das andere Auge zu applizieren. So werden eine Verdünnung des Farbstoffs im Tränenfilm und eine Aufnahme in Hornhaut und Bindehaut am anderen Auge und damit einhergehende Messungenauigkeiten vermieden.

Hauptprobleme bei der Tränenfilmaufrisszeit sind

- die deutliche Streuung der Werte und

- die geringe Reproduzierbarkeit.

Als Grund hierfür ist vor allem die fehlende Standardisierung bei der Fluoresceinapplikation zu nennen.

- Sollte man einen Streifen mit Fluorescein oder Tropfen verwenden?

- Tränkt man den Streifen mit Flüssigkeit, oder:

- Mit wie viel Flüssigkeit soll man ihn tränken?

- Wo und wie lange bringt man diesen Streifen ein?

Die Variable ist letztlich die Menge des eingebrachten Fluoresceins. So konnte bei Verwendung von $2 \mu \mathrm{l}$ oder weniger einer $5 \%$ Fluoresceinlösung eine höhere Reliabilität gezeigt werden als bei der Verwendung von Fluoresceinstreifen.

Bei Gesunden findet sich bei der Verwendung von üblichen Mengen Fluorescein (z. B. 1 Tropfen) eine Aufrisszeit von mehr als $10 \mathrm{~s}$ und entsprechend bei Patienten mit trockenem Auge eine Aufrisszeit von unter $10 \mathrm{~s}$.

Bei Verwendung einer Mikropipette finden sich entsprechend Werte von über bzw. unter $5 \mathrm{~s}$.

Die Sensitivität für die Erkennung eines trockenen Auges beträgt bei diesem Test $72 \%$ und die Spezifität $62 \%$ (Definition and Classification Subcommittee of the International Dry Eye WorkShop 2007; Jacobi et al. 2006; Tomlinson et al. 2011).

\section{nichtinvasive Break-up-Time (NIBUT)}

Als möglicher weiterer Nachteil der FBUT wird außerdem diskutiert, dass die Eingabe von Fluorescein an sich bereits die Physiologie des Tränenfilms stört. Als Alternative wurde daher die nichtinvasive Break-upTime (NIBUT) beschrieben. Hierbei wird ein Gitter oder ein anderes Muster auf die Hornhaut projiziert. Die NIBUT ist die Zeit vom letzten Lidschlag bis zur Veränderung des betrachteten Musters. Idealerweise wird das Bild bei der NIBUT mittels Kamera erfasst und durch einen Computer ausgewertet. 
Insgesamt zeigen sich bei der NIBUT höhere Werte als bei der FBUT, was eine Folge des angenommenen destabilisierenden Effekt des Fluoresceins auf den Tränenfilm sein könnte.

Die Methode erreicht eine Sensitivität von $82 \%$ und eine Spezifität von $86 \%$.

Die Normwerte streuen allerdings erheblich, je nach verwendetem Gerät (Tomlinson et al. 2011).

\section{Tränenfilmosmolarität}

Sowohl die erhöhte Verdunstung als auch die verminderte Produktion von Tränenflüssigkeit können zu einer Steigerung der Konzentration der Tränenflüssigkeit und damit zu einer Hyperosmolarität führen. Diese kann heute einfach und schnell im klinischen Alltag erfasst werden. Bei der Untersuchung wird das Osmometer an der Unterlidinnenseite in den Tränenfilm gesetzt. Durch Kapillarkräfte werden ca. 100 nl Tränenflüssigkeit auf einen Chip aufgenommen (Abb. 2). Der Osmolaritätswert wird innerhalb kürzester Zeit von dem Gerät angegeben.

Für jeden Patienten wird ein separater Chip verwendet, was zwar im Alltag sehr praktisch, aber auch kostenintensiv ist. Die Tränenosmolaritätswerte für normale Patienten liegen bei ca. 275-316 mOsms/l und die Methode erreicht bei einem Grenzwert von 316 mOsm/l eine Sensitivität von 59\% und eine Spezifität von 94\% (Tomlinson et al. 2011). Allerdings ist dieser Grenzwert bislang keineswegs fest etabliert.

Dennoch ist die Messung der Osmolarität bislang der beste Einzeltest zur Identifikation eines trockenen Auges, kann aber durch Kombination mehrerer Tests übertroffen werden.

\section{Interferometrie}

In der letzten Zeit entstanden verschiedene Verfahren, die, basierend auf dem Prinzip der Interferenz, die Lipidschicht des Tränenfilms quantifizieren wollen. Was Interferometrie ist, versteht man am besten, wenn man eine Pfütze auf der Straße betrachtet, in die ein Tropfen Motoröl gefallen ist. Bei Sonneneinstrahlung sieht man typische Lichtmuster auf der Pfütze. Diese Lichtmuster sind abhängig von der Dicke der Fettschicht und können daher auch verwendet werden, um diese Dicke zu quantifizieren. Während bislang meist nur eine semiquantitative Auswertung mittels Gradeinteilung gelingt, versprechen neuere Verfahren eine Angabe der Lipidschichtdicke in nm (Abb. 3; Tomlinson et al. 2011).

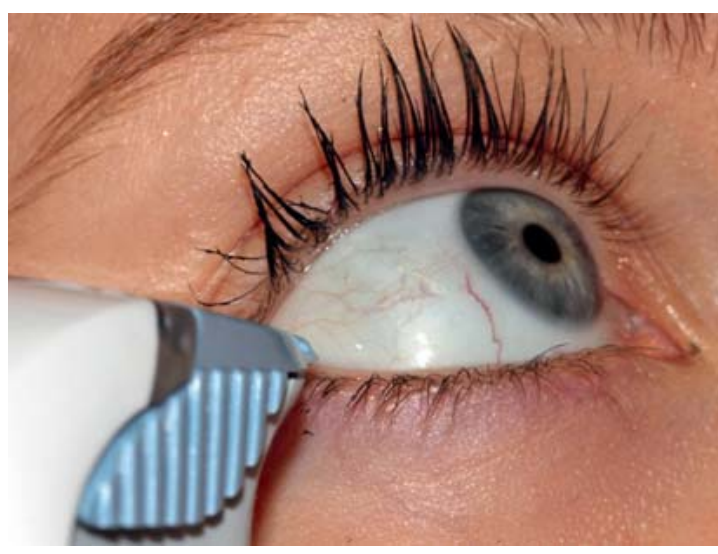

Abb. 2 Osmolaritätsmessung.
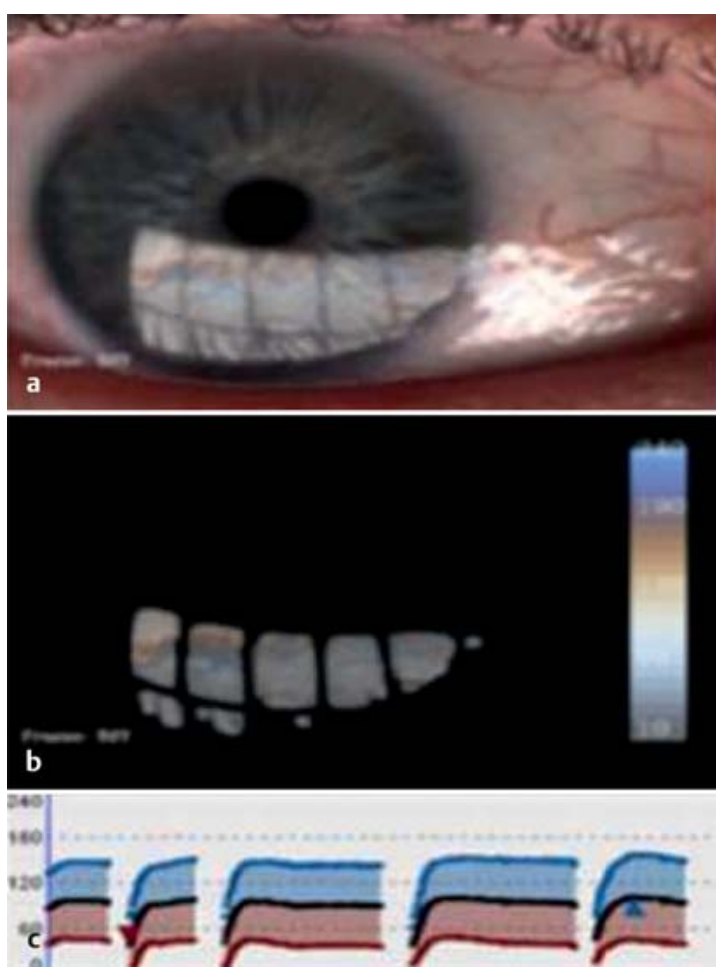

Abb. 3 Interferometrie. a Interferenzmuster auf der Augenoberfläche. b Subtraktionsbild (restliche Bildbestandteile herausgerechnet). c Analyse der Interferenzmuster mit Angabe eines Näherungswertes für die Lipidschichtdicke (hier etwa $90 \mathrm{~nm}$ ).

\section{Meibografie}

Mit der Meibografie lassen sich die Meibom-Drüsen in vivo darstellen, sodass eine Quantifizierung z.B. eines atrophiebedingten Funktionsausfalls ermöglicht wird. Während dies früher in Form einer transpalpebralen Diaphanoskopie am evertierten Lid erfolgte, existieren heute Infrarotkameras, die die Meibom-Drüsen direkt bildlich erfassen können (Abb.4). Die Methode wurde 

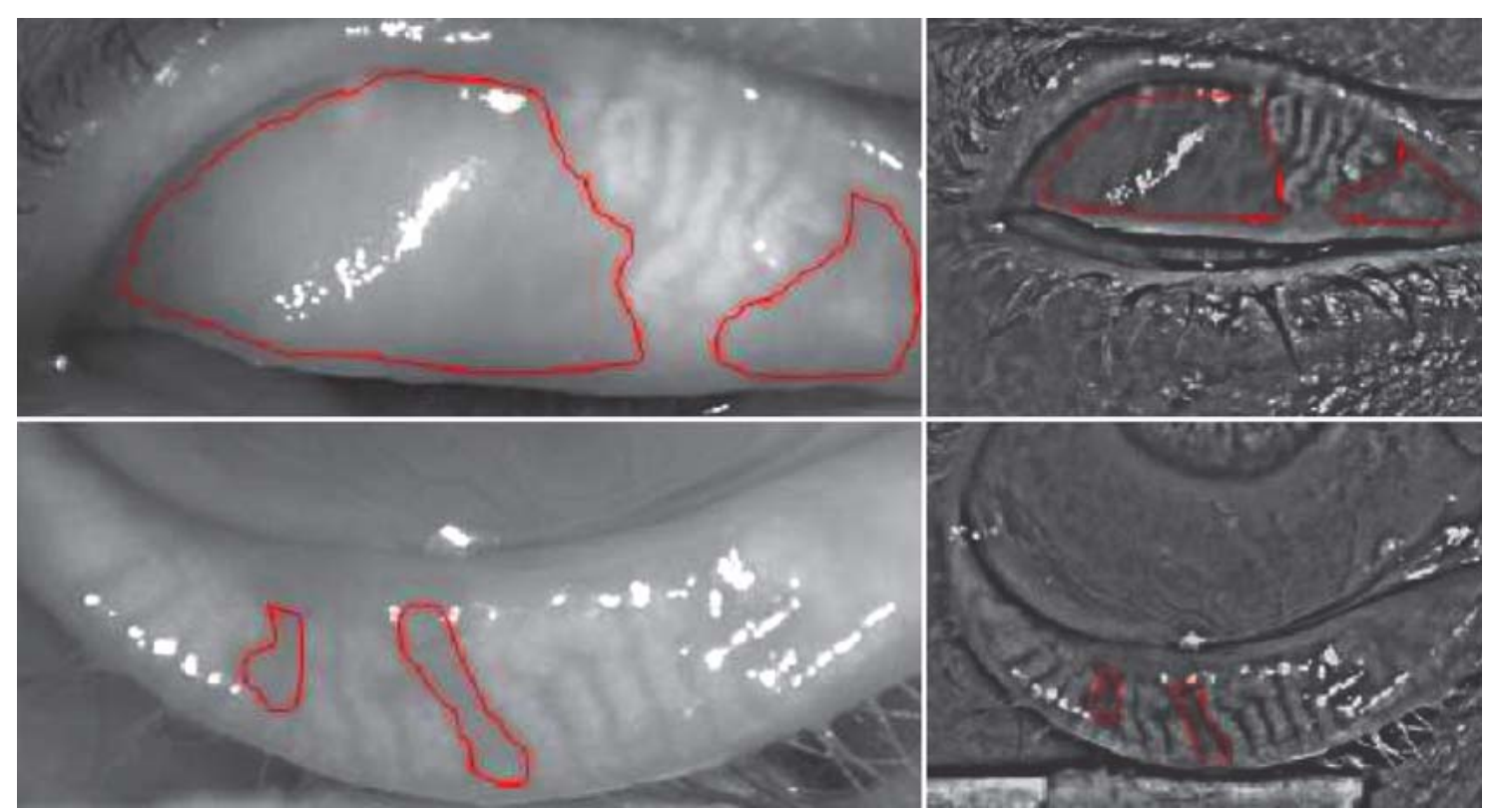

Abb. 4 Meibografie: Die atrophierten Drüsen sind rot umrandet markiert (aus Finis et al. 2012).

\section{Zielgerichtete Diagnostik}

Stellenwert der qualitativen Untersuchungen des Tränenfilms

- Standardtest für die qualitative Untersuchung des Tränenfilms ist die Messung der Tränenfilmaufrisszeit (Break-up-Time).

- Die Osmolaritätsmessung ist der beste Einzeltest zur Detektion eines trockenen Auges.

so deutlich patientenfreundlicher. Infrarotkameras, die mittels Software-Upgrade für die Meibografie nutzbar gemacht werden können, finden sich z.B. in Funduskameras oder Topografiesystemen.

Der Funktionsausfall kann mittels Software prozentual angegeben oder mittels verschiedener Gradeinteilungen semiquantitativ analysiert werden (Finis et al. 2012).

\section{Vitalfärbungen}

Zur Anfärbung der Hornhaut und Bindehaut stehen 3 Farbstoffe zur Verfügung (s. Infobox „Überblick“). Außerdem gibt es verschiedene Systeme zur Graduierung.

\section{Überblick}

Zur Verfügung stehende Farbstoffe zur Anfärbung der Hornhaut und Bindehaut

- Fluorescein

- Bengal-Rosa

- Lissamin-Grün

\section{Einteilung nach van Bijsterveld bzw. Bron et al.}

Sowohl nach van Bijsterveld (1969) als auch Bron et al. (2003) wird die Augenoberfläche eingeteilt in die

3 Bereiche

- Hornhaut,

- nasale Bindehaut und

- temporale Bindehaut.

Für jeden Bereich wird das Ausmaß der Anfärbung mit einer Punktzahl von 0-3 (van Bijsterveld 1969) oder 0-5 (Bron et al. 2003; Abb. 5) bewertet und ein Gesamtwert errechnet (van Bijsterveld 1969). 


\section{Graduierung des National Eye Institute}

Eine weitere Klassifikation ist die von Lemp (1995) publizierte Graduierung des National Eye Institute. Hier wird die Hornhaut in 5 Bereiche und die Bindehaut in $2 \times 3$ Bereiche eingeteilt. Für jeden Bereich wird ein Punktwert von 0-3 vergeben (Abb. 6).

\section{CLEK-Schema (Collaborative Longitudinal Evaluation of Keratoconus)}

Der Vollständigkeit halber sei an dieser Stelle noch das CLEK-Schema (Collaborative Longitudinal Evaluation of Keratoconus) erwähnt, bei dem die Bindehaut in 4 und die Hornhaut in 5 Areale aufgeteilt wird. Entscheidend ist hier vor allem das zentrale Hornhautareal, da eine verstärkte Anfärbbarkeit in diesem Bereich Hinweise auf eine Visusminderung liefern kann.

\section{Oxford-Schema}

Insgesamt präferieren wir die Beurteilung der Anfärbbarkeit nach dem Oxford-Schema. Dieses kann prinzipiell mit allen 3 Farbstoffen kombiniert werden.

Eine besonders häufig verwendete Variante ist die Kombination einer Fluoresceinfärbung für die Hornhaut und einer Lissamin-Grün-Färbung für die Bindehaut. Zur Vereinfachung ist aber auch die Beurteilung von Horn- und Bindehaut nur nach Fluoresceinapplikation möglich.

\section{Fluorescein}

Vorteile. Die Vorteile von Fluorescein liegen in

- der Verfügbarkeit und

- der guten Verträglichkeit.

Um die Fluoreszenz genau betrachten zu können, sollte der vordere Augenabschnitt mit einem Kobaltblaufilter im Bestrahlungsgang und möglichst einem gelben Sperrfilter im Beobachtungsstrahlengang betrachtet werden (Abb. 7).

Nachteile. Der große Nachteil des Fluoresceins ist die rasche Aufnahme des Farbstoffes in das Gewebe.

Durchführung. Es ist aufgrund der raschen Aufnahme des Farbstoffes in das Gewebe zwingend erforderlich,

\begin{tabular}{|l|c|l|}
\hline Abbildung & Grad & Kriterien \\
\hline A & 0 & $\begin{array}{l}\text { Anfärbung gleich oder geringer } \\
\text { als in Abbildung A }\end{array}$ \\
\hline B & I & $\begin{array}{l}\text { Anfärbung gleich oder geringer } \\
\text { als in Abbildung B, } \\
\text { größer als in Abbildung A }\end{array}$ \\
\hline C & II & $\begin{array}{l}\text { Anfärbung gleich oder geringer } \\
\text { als in Abbildung C, } \\
\text { größer als in Abbildung B }\end{array}$ \\
\hline D & III & $\begin{array}{l}\text { Anfärbung gleich oder geringer } \\
\text { als in Abbildung D, } \\
\text { größer als in Abbildung C }\end{array}$ \\
\hline E & IV & $\begin{array}{l}\text { Anfärbung gleich oder geringer } \\
\text { als in Abbildung E, } \\
\text { größer als in Abbildung D }\end{array}$ \\
\hline >E & V & \begin{tabular}{l} 
Anfärbung größer als in Abbildung E \\
\hline
\end{tabular} \\
\hline
\end{tabular}

Abb. 5 Oxford-Skala nach DEWS-Report 2007 (Definition and Classification Subcommittee of the International Dry Eye WorkShop 2007).
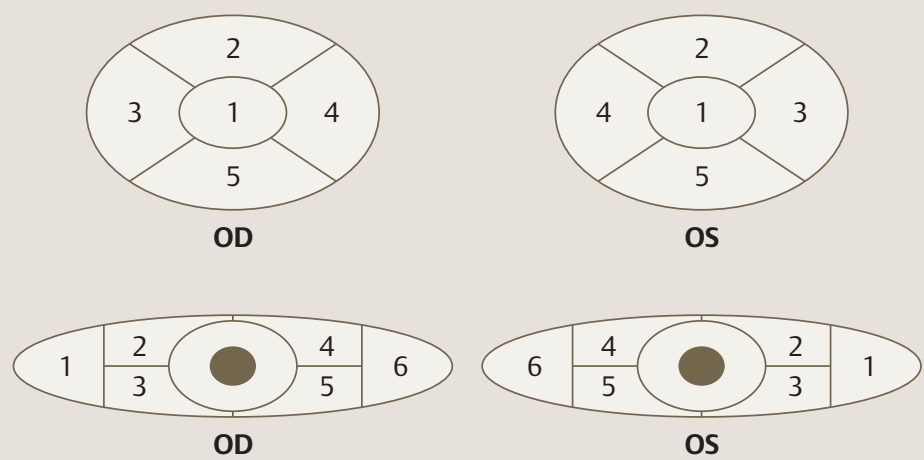

$\square$ Grad $0 \quad \therefore$ Grad 1

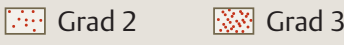

Abb. 6 National-Eye-Institute-Skala (nach Lemp 1995). OD = Oculus Dexter; OS = Oculus Sinister.

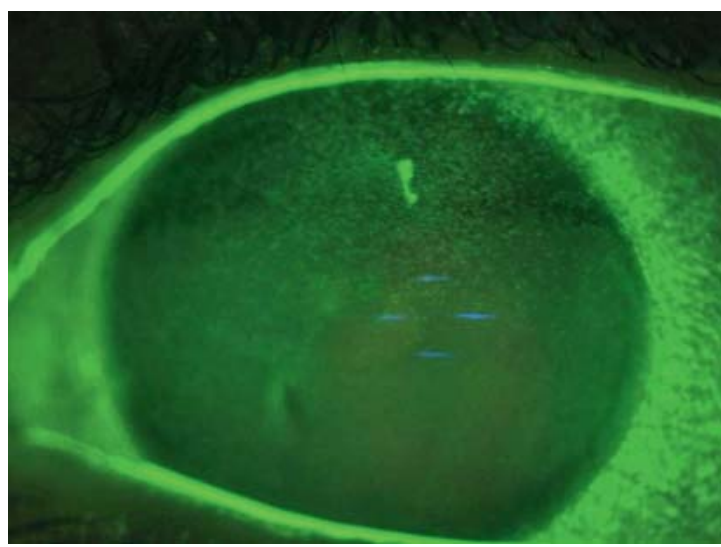

Abb. 7 Fluoresceinanfärbung von punktförmigen Epitheldefekten im Bereich der Hornhaut bei Keratoconjunctivitis sicca (Beleuchtung mit Kobaltblaufilter und Betrachtung mit Gelbfilter). 


\section{Prinzipien}

Untersuchungsprinzipien bei Fluoresceinfärbung

- Anfärbbarkeit (Staining) unmittelbar nach der Fluorescein-Break-up-Time (FBUT) bestimmen.

- Farbstoff zuerst nur in ein Auge und erst nach abgeschlossener Untersuchung Durchführung am zweiten Auge.

die Anfärbbarkeit (Staining) unmittelbar nach der Fluorescein-Break-up-Time (FBUT) zu bestimmen. Außerdem sollte der Farbstoff zuerst nur in ein Auge und erst nach abgeschlossener Betrachtung und Bewertung des Befunds in das zweite Auge gegeben werden. In klinischen Studien empfiehlt sich die Gabe von $2 \mu 12 \%$ Fluorescein mittels Mikropipette in den unteren Fornix und die Betrachtung des Stainings nach 2 Minuten.

\section{Bengal-Rosa}

Vorteile. Bengal-Rosa hat gegenüber Fluorescein den Vorteil, dass es insbesondere bei Anfärbung der Bindehaut einen besseren Kontrast gegenüber der Sklera ergibt (Abb. 8). Die Anfärbung der Hornhaut ist gut gegen eine blaue Iris zu erkennen, kann aber bei einer dunklen Iris schwierig sein. Der Kontrast kann durch Beleuchtung mit rotfreiem Licht verbessert werden.

Nachteile. Die Nachteile des Bengal-Rosa sind

- die Epitheltoxizität, die durch ultraviolette Strahlung, z. B. bei starker Sonnenlichtexposition, noch verstärkt wird, sowie

- die Reizung des Auges.

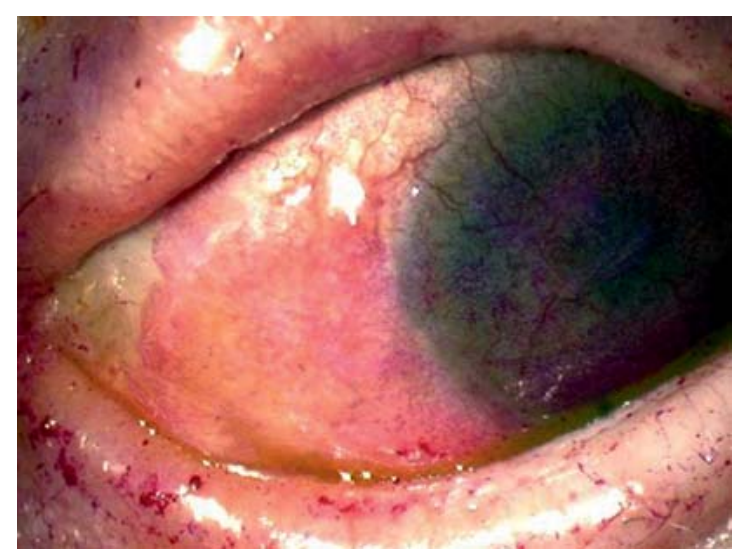

Abb. 8 Anfärbung von Defekt in der Muzinschicht, der Bindehaut und Hornhaut mittels Bengal-Rosa.
Sowohl der erwünschte Anfärbeeffekt als auch die unerwünschten Effekte wie Brennen und Schmerzen sind dosisabhängig.

Durchführung. Empfohlen wird die Applikation von $25 \mu \mathrm{l}$ 1\% Bengal-Rosa auf die obere Bindehaut bei Blick des Patienten nach unten und zurückgezogenem Lid. Häufig ist hierbei die Applikation von Lokalanästhetikum notwendig. Da der Farbstoff nicht in das Gewebe aufgenommen wird und auch nach längerer Zeit noch einen guten Kontrast ergibt, ist die Beurteilung nicht nach einer bestimmten Zeitvorgabe erforderlich. Wir empfehlen hier die Beurteilung nach 2 Minuten.

\section{- Lissamin-Grün}

Vorteile. Lissamin-Grün ist annähernd so gut verträglich wie Fluorescein und zeigt eine gute Kontrastierung bei der Anfärbung der Bindehaut, vergleichbar mit der von Bengal-Rosa. Die Färbung der Hornhaut ist bei blauer Iris gut zu erkennen, kann aber bei einer dunklen Iris schwierig abzugrenzen sein.

Durchführung. Der Kontrast kann durch Betrachtung mit einem Rotfilter verbessert werden. Empfohlen wird die Verwendung von $25 \mu$ 1 \% Lissamin-Grün (Definition and Classification Subcommittee of the International Dry Eye WorkShop 2007; Bron et al. 2003). Da auch Lissamin-Grün nicht in das Gewebe aufgenommen wird und auch nach längerer Zeit noch einen guten Kontrast ergibt, ist die Beurteilung nicht nach einer bestimmten Zeitvorgabe erforderlich. Wir empfehlen ebenfalls die Beurteilung nach $2 \mathrm{~min}$.

\section{Zielgerichtete Diagnostik}

Stellenwert der Vitalfärbungen

- Bei jedem Patienten mit Oberflächenerkrankung des Auges sollte eine Vitalfärbung mit anschließender Quantifizierung über eines der genannten Schemata erfolgen. 


\section{Ablauf der Diagnostik}

Grundsätzliche Überlegungen zeigt die Infobox „Prinzipien“. Der in Abb. 9 dargestellte Ablauf ist empfehlenswert (Tomlinson 2011).

\section{Prinzipien}

Grundsätze der Diagnostik des Tränenfilms und der Augenoberfläche

- Der Untersuchungsgang ist so zu strukturieren, dass sich die einzelnen Tests so wenig wie möglich gegenseitig beeinflussen.

- Invasive Tests sollten am Ende der Untersuchung erfolgen.

Interessenkonflikt: Die Autoren bestätigen, dass kein Interessenkonflikt vorliegt.

\section{Dieser Artikel erschien in den \\ Klinischen Monatsblättern für Augenheilkunde (DOI 10.1055/s-0033-1357966).}

\section{Quellenangaben}

1 Begley CG, Caffery B, Chalmers RL et al. Use of the dry eye questionnaire to measure symptoms of ocular irritation in patients with aqueous tear deficient dry eye. Cornea 2002; 21: 664-670

2 Bron AJ, Evans VE, Smith JA. Grading of corneal and conjunctival staining in the context of other dry eye tests. Cornea 2003; 22: $640-650$

3 Brewitt H, Zierhut M. Trockenes Auge: Anatomie, Physiologie, Pathophysiologie, Diagnostik, Therapie. Heidelberg: Kaden; 2001

4 Definition and Classification Subcommittee of the International Dry Eye WorkShop. The definition and classification of dry eye disease: report of the Definition and Classification Subcommittee of the International Dry Eye WorkShop (2007). Ocul Surf 2007; 5: 75-92

5 Finis D, Schrader S, Geerling G. Meibom-Drüsen-Dysfunktion. Klin Monatsbl Augenheilkd 2012; 229: 506-513

6 Finis D, Pischel N, König C et al. Vergleich des OSDI- und SPEEDFragebogens zur Evaluation des Sicca-Syndroms in der klinischen Praxis. Ophthalmologe 2014; 111: akzeptiert

7 Grubbs JR jr., Tolleson-Rinehart S, Huynh $\mathrm{K}$ et al. A review of quality of life measures in dry eye questionnaires. Cornea 2014; 33 : $215-218$

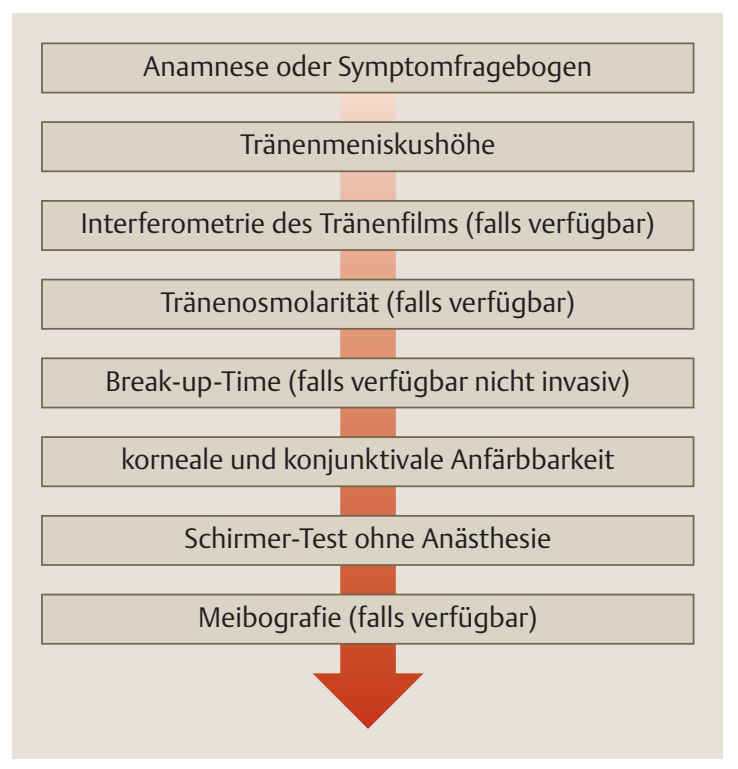

Abb. 9 Flussschema des Diagnostikablaufs.

8 Jacobi C, Dietrich T, Cursiefen C, Kruse FE. Das trockene Auge. Aktuelle Konzepte zu Klassifizierung, Diagnostik und Pathogenese. Ophthalmologe 2006; 103: 9-17

9 Lemp MA. Report of the National Eye Institute/Industry workshop on Clinical Trials in Dry Eyes. CLAO J 1995; 21: 221 - 232

10 Schiffman RM, Christianson MD, Jacobsen G et al. Reliability and validity of the Ocular Surface Disease Index. Arch Ophthalmol 2000; 118: 615-621

11 Tomlinson A, Bron AJ, Korb DR et al. The international workshop on Meibomian gland dysfunction: report of the diagnosis subcommittee. Invest Ophthalmol Vis Sci 2011; 52: 2006-2049

12 van Bijsterveld OP. Diagnostic tests in the Sicca syndrome. Arch Ophthalmol 1969; 82: 10-14

13 Yokoi N, Bron AJ, Tiffany JM et al. Relationship between tear volume and tear meniscus curvature. Arch Ophthalmol 2004; 122: $1265-1269$

\section{Korrespondenzadresse}

Univ.-Prof. Dr. Dr. Stefan Schrader

Universitätsaugenklinik

Heinrich-Heine-Universität

Moorenstraße 5

40225 Düsseldorf

Telefon: $0211 / 81-16041$

Fax: $0211 / 81-16298$

E-Mail: stefan.schrader@med.uni-duesseldorf.de 


\section{CME.thieme.de}

\section{CME-Fragen}

\section{CME-Teilnahme}

- Viel Erfolg bei Ihrer CME-Teilnahme unter http://cme.thieme.de

- Bitte informieren Sie sich über die genaue Gültigkeitsdauer unter http://cme.thieme.de

- Sollten Sie Fragen zur Online-Teilnahme haben, unter http://cme.thieme.de/hilfe finden Sie eine ausführliche Anleitung.

\section{1}

Eine der Aussagen zu Symptomfragebögen ist nicht korrekt. Welche?
A Ein strukturierter Symptomfragebogen bietet eine gute Möglichkeit zum Screening von Patienten mit potenziellen Oberflächenerkrankungen des Auges.

B Der Fragebogen des Ressorts „trockenes Auge“ vom BVA eignet sich sehr gut zur Erfassung der Anamnese.

C Für klinische Studien empfiehlt sich die Verwendung der etablierten OSDI- oder DEQ-Fragebögen, vor allem aufgrund ihrer guten Auflösung.

D Der IDEEL-Fragebogen scheint in der Erfassung von Auswirkungen des trockenen Auges auf die Lebensqualität dem OSDI überlegen zu sein.

E Der DEQ (Dry Eye Questionnaire) ist ein innovatives Messinstrument für die Anamneseerfassung bei trockenem Auge, das seine Wertigkeit erst zeigen muss.

\section{2}

Welche Aussage zum

Schirmer-II-Test ist zutreffend?
A Der Schirmer-II-Test wird immer ohne Lokalanästhesie durchgeführt.
B Der Schirmer-II-Test soll die Reizsekretion der Tränendrüse widerspiegeln.
C Durchschnittswerte von Männern und Frauen unterscheiden sich signifikant.
D Beim Schirmer-II-Test liegen die Werte ca. 40\% niedriger im Vergleich zum Schirmer-I-Test.
E Das Ergebnis wird nach 10 Minuten abgelesen.

3 Nur eine der folgenden Aussage zur Meniskometrie trifft zu. Welche?
A Die Höhe des Tränenmeniskus am Ober- und Unterlid gibt direkt an, wie viel Tränenflüssigkeit beim nächsten Lidschlag zur Verfügung steht.
B Die Höhe des Tränenmeniskus steigt nicht nach Applikation von Augentropfen oder Einbringen von Punctum-Okklusiven.
C Werte über $0,2 \mathrm{~mm}$ gelten als pathologisch.
D Die Zugabe von Fluorescein führt nicht zu einer Beeinflussung des Messergebnisses.
E Die Höhe des Tränenmeniskus korreliert nicht mit dem Schirmer-Test.

4

Wodurch ist die Tränenfilmaufrisszeit (Break-up-Time) gekennzeichnet?
A Die Tränenfilmaufrisszeit (Break-up-time) ist der klassische Test zur Diagnose eines quantitativen Tränenmangels.

B Zur Messung wird klassischerweise Bengal-Rosa in den unteren Fornix eingebracht.

C Die Sensitivität der Tränenfilmaufrisszeit für die Erkennung eines trockenen Auges liegt bei ca. $90 \%$.

D Die Tränenfilmaufrisszeit ist unabhängig von der eingegebenen Fluoresceinmenge.

E Bei Gesunden findet sich bei der Verwendung von üblichen Mengen Fluorescein (z. B. 1 Tropfen) eine Aufrisszeit von mehr als 10 Sekunden. 


\section{CME-Fragen}

\section{5}

Eine der folgenden Aussagen zur Tränenfilmosmolarität trifft nicht zu. Welche?
A Sowohl die erhöhte Verdunstung als auch die verminderte Produktion von Tränenflüssigkeit können zu einer Hyperosmolarität führen.

B Die Messung der Osmolarität eignet sich zur Diagnosestellung bei der Meibom-DrüsenDysfunktion.

C Die Messung der Osmolarität mittels Osmometer erreicht bei einem Cut-off von $316 \mathrm{mOsm} / \mathrm{l}$ eine Sensitivität von $59 \%$ und eine Spezifität von $94 \%$.

D Die Messung der Osmolarität ist bislang der beste Einzeltest zur Identifikation eines trockenen Auges.

E Das Osmometer wird bei der Untersuchung an der Unterlidinnenseite in den Tränenfilm gesetzt, und durch Kapillarkräfte werden ca. 100 nl Tränenflüssigkeit auf einen Chip aufgenommen.
6

Welche Aussage zur Meibografie ist richtig?
A Sie basiert auf dem Prinzip der Interferometrie.

B Sie kann nur in Narkose durchgeführt werden.

C Sie dient zur Testung der Meibom-Drüsen-Funktion.

D Sie kann zur Darstellung der Tränendrüse genutzt werden.

E Mit ihr lassen sich die Meibom-Drüsen in vivo darstellen, sodass z. B. eine Quantifizierung eines atrophiebedingten Ausfalls ermöglicht wird.

\section{7}

Die Vorteile von Fluorescein liegen in ...
A ... der raschen Aufnahme des Farbstoffes in das Gewebe.

B ... der guten Verträglichkeit.

C ... der Spezifität für die Bindehaut.

D ... der Anfärbung von Muzin.

E ... der Darstellung von kornealen Stromanarben.

\section{8}

Welche Aussage zu Graduierungssystemen für die Augenoberfläche trifft zu?
A Sowohl beim System nach van Bijsterveld als auch Bron et al. wird nur die Hornhautoberfläche bewertet.

B Die von Lemp publizierte Graduierung des National Eye Institute bezieht auch die Lidkanten mit in die Bewertung ein.

C Die Beurteilung der Anfärbbarkeit nach dem Oxford-Schema sollte nur nach Anfärbung mit Bengal-Rosa durchgeführt werden.

D Bei dem CLEK-Schema wird das zentrale Hornhautareal vernachlässigt.

E Eine besonders häufig verwendete Variante der Augenoberflächenanfärbung zur Bewertung mittels Oxford-Schema ist die Kombination einer Fluoresceinfärbung für die Hornhaut und einer Lissamin-Grün-Färbung für die Bindehaut. 


\section{CME-Fragen}

Diagnostik des Tränenfilms und der Augenoberfläche

\section{9}

Eine der folgenden Aussagen zu Bengal-Rosa trifft nicht zu. Welche?
A Empfohlen wird die Applikation von $25 \mu \mathrm{l}$ 1\% Bengal-Rosa auf die obere Bindehaut bei Blick des Patienten nach unten und zurückgezogenem Lid.

B Ein Vorteil von Bengal-Rosa ist die geringe Epitheltoxizität.

C Der erwünschte Anfärbeeffekt, aber auch die unerwünschten Effekte - Brennen und Schmerzen sind dosisabhängig.

D Die Beurteilung nach einer bestimmten Zeitvorgabe ist nicht erforderlich, da der Farbstoff nicht in das Gewebe aufgenommen wird und auch nach längerer Zeit noch einen guten Kontrast liefert.

E Bengal-Rosa hat gegenüber Fluorescein den Vorteil, dass es insbesondere bei Anfärbung der Bindehaut einen besseren Kontrast gegenüber der Sklera ergibt.

\section{0}

Wodurch ist die Vitalfärbung mit Lissamin-Grün gekennzeichnet?
A Lissamin-Grün ist im Vergleich zu Fluorescein deutlich schlechter verträglich.

B Lissamin-Grün zeigt eine gute Kontrastierung bei der Anfärbung der Bindehaut, vergleichbar mit der von Bengal-Rosa.

C Die sofortige Beurteilung ist erforderlich, da Lissamin-Grün schnell in das Gewebe aufgenommen wird.

D Der Kontrast kann durch Betrachtung mit einem Grünfilter verbessert werden.

E Lissamin-Grün kann zu dauerhafter Grünfärbung der Iris führen. 